PROCEEDINGS OF THE

AMERICAN MATHEMATICAL SOCIETY

Volume 130, Number 3, Pages 727-731

S 0002-9939(01)06150-0

Article electronically published on June 21, 2001

\title{
FINITE DIMENSIONAL REPRESENTATIONS OF THE SOFT TORUS
}

\author{
SØREN EILERS AND RUY EXEL \\ (Communicated by David R. Larson)
}

\begin{abstract}
The soft tori constitute a continuous deformation, in a very precise sense, from the commutative $C^{*}$-algebra $C\left(\mathbb{T}^{2}\right)$ to the highly non-commutative $C^{*}$-algebra $C^{*}\left(\mathbb{F}_{2}\right)$. Since both of these $C^{*}$-algebras are known to have a separating family of finite dimensional representations, it is natural to ask whether that is also the case for the soft tori. We show that this is in fact the case.
\end{abstract}

\section{INTRODUCTION}

Knowing that a given $C^{*}$-algebra has many representations on finite dimensional Hilbert spaces is of great importance to understanding structural properties of it. Among $C^{*}$-algebras, those who possess a separating family of finite dimensional representations are called residually finite dimensional or just $R F D$. This class was studied in [12], 11] and [1, and more recent insight about it has lead to important advances in classification theory and the theory of quasidiagonal $C^{*}$-algebras (see, e.g., [2], [5] and [6]).

For any $\varepsilon \geq 0$ we define a $C^{*}$-algebra $A_{\varepsilon}$ as the universal (unital) $C^{*}$-algebra defined by the generators $u, v$ subject to the relations

$$
u u^{*}=u^{*} u=1, \quad v v^{*}=v^{*} v=1, \quad\|u v-v u\| \leq \varepsilon .
$$

As recorded in [8, $A_{0}$ is the commutative $C^{*}$-algebra of functions over the torus $\mathbb{T}^{2}$, and $A_{\varepsilon}$ is the full $C^{*}$-algebra of the free group of two generators $\mathbb{F}_{2}$ whenever $\varepsilon \geq 2$. For $\varepsilon$ between 0 and 2 we get a class of $C^{*}$-algebras which are commonly referred to as soft tori. These $C^{*}$-algebras are of relevance to several problems in operator algebra theory (see [10]) and have been extensively studied in [3], [8], [9], [7].

The starting point of the investigation reported on in the present paper is a result from [9], stating that the soft tori form a continuous field interpolating between the (hard) torus and the group $C^{*}$-algebra of the free group. Since $C\left(\mathbb{T}^{2}\right)$ is obviously RFD, and since $C^{*}\left(\mathbb{F}_{2}\right)$ was proved to be RFD in 4 - a surprise at the time - we are naturally led to the question of whether the same is true for the interpolating family $A_{\varepsilon}$. We are going to prove that this is the case.

Received by the editors November 19, 1998 and, in revised form, August 28, 2000.

2000 Mathematics Subject Classification. Primary 46L05; Secondary 46L65, 46L85, 47B20.

This work was partially supported by the Carlsberg Foundation.

(C)2001 American Mathematical Society 


\section{Methods}

We prove that $A_{\varepsilon}$ is RFD using an adaptation of the compression argument developed by Choi in [4] to prove that $C^{*}\left(\mathbb{F}_{2}\right)$ is RFD. However, Choi's argument does not apply directly to $A_{\varepsilon}$ since one cannot arrange for the compressions to satisfy the commutation relation. We instead argue via an auxiliary $C^{*}$-algebra, thus employing a method from [3] and [8] which lies behind many results about the structural properties of $A_{\varepsilon}$.

We define $B_{\varepsilon}$ as the universal $C^{*}$-algebra given by the generators $\left\{u_{n}\right\}_{n \in \mathbb{Z}}$ and the relations

$$
u_{n} u_{n}^{*}=u_{n}^{*} u_{n}=1, \quad\left\|u_{n+1}-u_{n}\right\| \leq \varepsilon .
$$

Clearly one can define an automorphism $\alpha$ on $B_{\varepsilon}$ by

$$
u_{n} \mapsto u_{n+1},
$$

and as seen in [8] one has

$$
A_{\varepsilon}=B_{\varepsilon} \rtimes_{\alpha} \mathbb{Z}
$$

There is a faithful conditional expectation $E_{\alpha}: A_{\varepsilon} \longrightarrow B_{\varepsilon}$.

Our strategy will be to prove that $A_{\varepsilon}$ is RFD by proving that $B_{\varepsilon}$ is RFD in a way which is covariant with $\alpha$.

\section{Finite dimensional Representations of $B_{\varepsilon}$}

We start out by finding a new picture of $B_{\varepsilon}$ by generators and relations.

Lemma 3.1. For any $\varepsilon<2, B_{\varepsilon}$ is isomorphic to the universal $C^{*}$-algebra generated by $v_{0},\left\{h_{n}\right\}_{n \in \mathbb{Z}}$ subject to the relations

$$
v_{0} v_{0}^{*}=v_{0}^{*} v_{0}=1, \quad h_{n}=h_{n}^{*}, \quad\left\|h_{n}\right\| \leq \frac{2}{\pi} \arcsin (\varepsilon / 2) .
$$

Proof. Let us denote the $C^{*}$-algebra generated by $v_{0}$ and $h_{n}$ subject to (2) by $B_{\varepsilon}^{\prime}$. We can define a map $\varphi: B_{\varepsilon} \longrightarrow B_{\varepsilon}^{\prime}$ by

$$
u_{n} \mapsto \begin{cases}e^{i \pi h_{n}} \cdots e^{i \pi h_{1}} v_{0}, & n>0, \\ v_{0}, & n=0, \\ e^{-i \pi h_{n}} \cdots e^{-i \pi h_{-1}} v_{0}, & n<0,\end{cases}
$$

since the elements to the right of the arrow above satisfy the relations (1). Similarly, the universal property of $B_{\varepsilon}^{\prime}$ allows for a map $\psi: B_{\varepsilon}^{\prime} \longrightarrow B_{\varepsilon}$ defined by

$$
v_{0} \mapsto u_{0}, \quad h_{n} \mapsto \frac{1}{i \pi} \log \left(u_{n} u_{n-1}^{*}\right) .
$$

Clearly $\varphi$ and $\psi$ are each others' inverse.

This characterization can be used to shorten the proof of [8, 2.2], stating that $B_{\varepsilon}$ is homotopic to $C(\mathbb{T})$. To see this, define maps $\varphi: B_{\varepsilon}^{\prime} \longrightarrow C(\mathbb{T})$ and $\psi:$ $C(\mathbb{T}) \longrightarrow B_{\varepsilon}^{\prime}$ by the correspondence $v_{0} \leftrightarrow[z \mapsto z], h_{n} \leftrightarrow 0$. Clearly $\varphi \psi=\operatorname{id}_{C(\mathbb{T})}$, and $\chi_{t}: B_{\varepsilon}^{\prime} \longrightarrow B_{\varepsilon}^{\prime}$ given by

$$
\chi_{t}\left(v_{0}\right)=v_{0}, \quad \chi_{t}\left(h_{n}\right)=t h_{n}
$$

provides a homotopy from $\operatorname{id}_{B_{\varepsilon}^{\prime}}$ to $\psi \varphi$.

In the following proof, we denote by $\operatorname{Alg}(X)$ the smallest $*$-algebra, not necessarily closed, generated by the set $X$ inside some $C^{*}$-algebra. 
Proposition 3.2. For any $\varepsilon<2, B_{\varepsilon}$ is RFD. In fact, for any $0 \neq b \in B_{\varepsilon}$ there exists $n \in \mathbb{N}$, an automorphism $\beta$ of $\mathbf{M}_{n}$ and a representation $\rho: B_{\varepsilon} \longrightarrow \mathbf{M}_{n}$ with the properties

$$
\rho(b) \neq 0, \quad \beta \rho=\rho \alpha .
$$

Proof. For the first claim we use the characterization of $B_{\varepsilon}$ given by the relations in (2) and proceed as in [4]. Fix a faithful non-degenerate representation $\pi: B_{\varepsilon} \longrightarrow$ $\mathbb{B}(\mathcal{H})$, where we may assume that $\mathcal{H}$ is a separable Hilbert space.

Let $P_{m}$ be a sequence of projections, with $\operatorname{rank}\left(P_{m}\right)=m$, converging strongly to the unit of $\mathbb{B}(\mathcal{H})$, and abbreviate

$$
T_{0, m}=P_{m} \pi\left(v_{0}\right) P_{m}, \quad K_{n, m}=P_{m} \pi\left(h_{n}\right) P_{m} .
$$

Now note that for each $m$ the collection of elements $\left\{V_{0, m}, H_{n, m}: n \in \mathbb{Z}\right\}$ defined by

$$
\begin{gathered}
V_{0, m}=\left[\begin{array}{cc}
T_{0, m} & \sqrt{P_{m}-T_{0, m} T_{0, m}^{*}} \\
\sqrt{P_{m}-T_{0, m}^{*} T_{0, m}} & -T_{0, m}^{*}
\end{array}\right], \\
H_{n, m}=\left[\begin{array}{cc}
K_{n, m} & 0 \\
0 & K_{n, m}
\end{array}\right]
\end{gathered}
$$

satisfies (2) in $\mathbf{M}_{2}\left(P_{m} \mathbb{B}(\mathcal{H}) P_{m}\right) \simeq \mathbf{M}_{2 m}$. Consequently we get representations $\pi_{m}: B_{\varepsilon} \longrightarrow \mathbf{M}_{2 m}$. We are going to check, following [4], that

$$
\underline{\pi}: B_{\varepsilon} \longrightarrow \prod_{m=1}^{\infty} \mathbf{M}_{2 m}, \quad \underline{\pi}(b)=\left(\pi_{m}(b)\right)_{m=1}^{\infty}
$$

is an isometry. It suffices to check that $\|\underline{\pi}(x)\| \geq\|x\|-\eta$ for any $\eta>0$ and any $x \in \operatorname{Alg}\left(\left\{v_{0}, h_{-N}, \ldots, h_{N}\right\}\right)$. Fix $\eta, N$ and $x$ and write

$$
x=F\left(v_{0}, h_{-N}, \ldots, h_{N}\right)
$$

where $F$ is some finite linear combination of finite words in $2 N+2$ variables and their adjoints. Since, when $m$ goes to infinity,

$$
V_{0, m} \longrightarrow\left[\begin{array}{cc}
\pi\left(v_{0}\right) & 0 \\
0 & -\pi\left(v_{0}\right)^{*}
\end{array}\right], \quad H_{n, m} \longrightarrow\left[\begin{array}{cc}
\pi\left(h_{n}\right) & 0 \\
0 & \pi\left(h_{n}\right)
\end{array}\right],
$$

strongly in the unit ball of $\mathbf{M}_{2}(\mathbb{B}(\mathcal{H}))$ we conclude that

$$
\begin{aligned}
& \limsup _{m \longrightarrow \infty}\left\|F\left(V_{0, m}, H_{-N, m}, \ldots, H_{N, m}\right)\right\| \\
& \geq\left\|\lim _{m \longrightarrow \infty} F\left(V_{0, m}, H_{-N, m}, \ldots, H_{N, m}\right)\right\| \\
& =\left\|\left[\begin{array}{cc}
\pi\left(F\left(v_{0}, h_{-N}, \ldots, h_{N}\right)\right) & 0 \\
0 & \pi\left(F\left(-v_{0}^{*}, h_{-N}, \ldots, h_{N}\right)\right)
\end{array}\right]\right\| \\
& \geq\|\pi(x)\|=\|x\| \text {. }
\end{aligned}
$$

We can hence find $m$ such that

$$
\|\underline{\pi}(x)\| \geq\left\|\pi_{m}(x)\right\|=\left\|F\left(V_{0, m}, H_{-N, m}, \ldots, H_{N, m}\right)\right\| \geq\|x\|-\eta .
$$

For the second claim, we go back to the original presentation (1) of $B_{\varepsilon}$ by unitary generators only. For a given $b \in B_{\varepsilon}$ with $\|b\|=1$ we fix, using the first part of the proposition, a finite dimensional representation $\pi: B_{\varepsilon} \longrightarrow \mathbf{M}_{m}$ with $\|\pi(b)\|>\frac{3}{4}$. We also fix $c$ and $N \in \mathbb{N}$ such that

$$
\|b-c\|<\frac{1}{4}, \quad c \in \operatorname{Alg}\left(u_{-N}, \ldots, u_{N}\right) .
$$


Choose $M>0$ and unitaries $v_{0}^{ \pm}, \ldots, v_{M}^{ \pm} \in \mathbf{M}_{m}$ with the properties

$$
\left\|v_{n+1}^{ \pm}-v_{n}^{ \pm}\right\| \leq \varepsilon, \quad v_{0}^{ \pm}=\pi\left(u_{ \pm N}\right), \quad v_{M}^{ \pm}=1 .
$$

There is then exactly one representation $\pi^{\prime}: B_{\varepsilon} \longrightarrow \mathbf{M}_{m}$ which is $2(N+M)$ periodic in the sense that $\pi^{\prime}\left(u_{n}\right)=\pi^{\prime}\left(u_{n+2(N+M)}\right)$ and satisfies

$$
\pi^{\prime}\left(u_{n}\right)= \begin{cases}v_{-N-n}^{-}, & -M-N \leq n<-N, \\ \pi\left(u_{n}\right), & -N \leq n \leq N \\ v_{n-N}^{+}, & N<n \leq N+M .\end{cases}
$$

Note that $\pi^{\prime}(c)=\pi(c)$; in particular $\left\|\pi^{\prime}(c)\right\| \geq \frac{1}{2}$.

Now let $n=2(N+M) m$. With $\beta$ defined as the backward cyclic shift in block form (with period $2(N+M)$ ) we may define a covariant representation $\rho$ of $B_{\varepsilon}$ on $\mathbf{M}_{n}$ by

$$
u_{i} \mapsto\left[\begin{array}{llll}
\pi^{\prime}\left(u_{i}\right) & & & \\
& \pi^{\prime}\left(u_{i+1}\right) & & \\
& & \ddots & \\
& & & \pi^{\prime}\left(u_{i+2(N+M)-1}\right)
\end{array}\right]
$$

We have

$$
\|\rho(b)\| \geq\left\|\pi^{\prime}(b)\right\| \geq\left\|\pi^{\prime}(c)\right\|-\frac{1}{4}>0
$$

\section{Finite dimensional Representations of $A_{\varepsilon}$}

Theorem 4.1. For any $\varepsilon>0$, let $A_{\varepsilon}$ be the universal $C^{*}$-algebra generated by a pair of unitaries subject to the relation $\|u v-v u\| \leq \varepsilon$. Then $A_{\varepsilon}$ is residually finite dimensional in the sense that it admits a separating family of finite dimensional representations.

Proof. We may assume that $0<\varepsilon<2$. Let $0 \neq a \in A_{\varepsilon}$. Then also $b=E_{\alpha}\left(a^{*} a\right)$ is nonzero, for the conditional expectation is faithful. Choose $n, \rho$ and $\beta$ as in Proposition 3.2 and define

$$
\pi: A_{\varepsilon}=B_{\varepsilon} \rtimes_{\alpha} \mathbb{Z} \longrightarrow \mathbf{M}_{n} \rtimes_{\beta} \mathbb{Z}
$$

as the extension to the crossed product of the covariant $*$-homomorphism $\rho$. We then have, with $E_{\beta}$ the conditional expectation from $\mathbf{M}_{n} \rtimes_{\beta} \mathbb{Z}$ to $\mathbf{M}_{n}$,

$$
E_{\beta}\left(\pi\left(a^{*} a\right)\right)=\pi\left(E_{\alpha}\left(a^{*} a\right)\right)=\rho(b) \neq 0,
$$

so $\pi(a) \neq 0$.

Note finally that since $\beta$ is inner,

$$
\mathbf{M}_{n} \rtimes_{\beta} \mathbb{Z}=\mathbf{M}_{n} \rtimes_{\text {id }} \mathbb{Z} \simeq C(\mathbb{T}) \otimes \mathbf{M}_{n} .
$$

Therefore we may compose $\pi$ with an evaluation map of $C(\mathbb{T}) \otimes \mathbf{M}_{n}$ to exhibit an $n$-dimensional representation which does not vanish on $a$.

Linear algebra tells us that $\mathbf{M}_{n}$ has a faithful tracial state and has the property that every matrix $x$ which is hyponormal is the sense that

$$
x^{*} x \geq x x^{*}
$$

is in fact normal. As in [4], we may conclude: 
Corollary 4.2. For any $\varepsilon, A_{\varepsilon}$ has a faithful tracial state, and any hyponormal operator in $A_{\varepsilon}$ is normal.

Proof. Such properties clearly pass from matrices to sums of the form $\prod_{n \in \mathbb{N}} \mathbf{M}_{m_{n}}$, and from these sums to any of their subalgebras. By the theorem, $A_{\varepsilon}$ is one such.

\section{REFERENCES}

[1] R. J. Archbold, On residually finite-dimensional $C^{*}$-algebras, Proc. Amer. Math. Soc. 123 (1995), no. 9, 2935-2937. MR 95m:46089

[2] B. Blackadar and E. Kirchberg, Generalized inductive limits of finite-dimensional $C^{*}$ algebras, Math. Ann. 307 (1997), no. 3, 343-380. MR 98c:46112

[3] C. Cerri, Non-commutative deformations of $C\left(\mathbb{T}^{2}\right)$ and $K$-theory, Internat. J. Math. 8 (1997), no. 5, 555-571. MR 98j:46082

[4] M.D. Choi, The full $C^{*}$-algebra of the free group on two generators, Pacific J. Math. 87 (1980), no. 1, 41-48. MR 82b:46069

[5] M. Dădărlat, Nonnuclear subalgebras of AF algebras, Amer. J. Math. 122 (2000), no. 3, 581-597. CMP 2000:13

[6] $\longrightarrow$ On the approximation of quasidiagonal $C^{*}$-algebras, J. Funct. Anal 167 (1999), no. 1, 69-78. MR 2000f:46069

[7] G.A. Elliott, R. Exel, and T.A. Loring, The soft torus. III. The flip, J. Operator Theory 26 (1991), no. 2, 333-344. MR 94f:46086

[8] R. Exel, The soft torus and applications to almost commuting matrices, Pacific J. Math. 160 (1993), 207-217. MR 94f:46091

[9] R. Exel, The soft torus: a variational analysis of commutator norms, J. Funct. Anal. 126 (1994), no. 2, 259-273. MR 95i:46085

[10] R. Exel and T.A. Loring, Invariants of almost commuting unitaries, J. Funct. Anal. 95 (1991), 364-376. MR 92a:46083

[11] _ Finite-dimensional representations of free product $C^{*}$-algebras, Internat. J. Math. 3 (1992), 469-476. MR 93f:46091

[12] K. R. Goodearl and P. Menal, Free and residually finite-dimensional $C^{*}$-algebras, J. Funct. Anal. 90 (1990), no. 2, 391-410. MR 91f:46078

Matematisk Afdeling, Københavns Universitet, Universitetsparken 5, DK-2100 CopenHAGEN $\varnothing$, DENMARK

E-mail address: eilers@math.ku.dk

$U R L:$ http://www.math.ku.dk/ eilers

Departamento de Matemática, Universidade Federal de Santa Catarina, 88040-900 Florianópolis SC, BRAZIL

E-mail address: exel@mtm.ufsc.br

$U R L:$ http://www.mtm.ufsc.br/ ${ }^{\sim e x e l}$ 